\title{
Envelope demodulation method based on SET for fault diagnosis of rolling bearings under variable speed
}

\author{
Zengqiang MA***, Xin LI***, Suyan LIU***, Yongjie GE**** and Feiyu LU** \\ * State Key Laboratory of Mechanical Behavior and System Safety of Traffic Engineering Structures, Shijiazhuang Tiedao University \\ 17 North 2nd Ring Road East, Shijiazhuang, Hebei Province 050043, China \\ ** School of Electrical and Electronic Engineering, Shijiazhuang Tiedao University \\ 17 North 2nd Ring Road East, Shijiazhuang, Hebei Province 050043, China \\ *** School of Traffic and Transportation, Shijiazhuang Tiedao University \\ 17 North 2nd Ring Road East, Shijiazhuang, Hebei Province 050043, China \\ E-mail: 972421932@qq.com \\ ${ }^{* \star * \star}$ College of Electronic and Information Engineering, Southwest University \\ 2 Tiansheng Road, Beibei District, Chongqing 400715, China
}

Received: 15 July 2020; Revised: 9 September 2020; Accepted: 27 October 2020

\begin{abstract}
Time-frequency analysis can effectively reveal the fault characteristics of rolling bearings under variable speed conditions. However, the traditional time-frequency analysis method cannot meet the requirements of anti-noise ability and time-frequency concentration. The synchroextracting transform (SET) as a novel time-frequency analysis method makes up for these defects, so the envelope demodulation time-frequency analysis method based on SET for fault diagnosis is proposed. Firstly, the best resonant band is selected by the adaptive band-pass filter based on fast spectral kurtosis. Then, the amplitude envelope of filtered signal is calculated by the Hilbert transform. Finally, SET is applied to analyze the amplitude envelope, and the fault information can be extracted from the envelope demodulation spectrum. The simulation signal and experimental signal are analyzed respectively. The results prove the effectiveness of the method. Compared with the short-time Fourier transform (STFT) and synchrosqueezing transform (SST), the proposed method is more prominent in the anti-noise property and time-frequency concentration. In addition, the performance in case of the band-pass filter failure is tested. The results show that the proposed method is still valid, and has excellent robustness and self-adaptability. Therefore, the proposed method shows significant application prospects and promotion value for fault diagnosis of variable-speed rolling bearing.
\end{abstract}

Keywords : Rolling bearings, Fault diagnosis, Synchroextracting transform, Envelope demodulation, Variable speed

\section{Introduction}

Rolling bearing is a crucial component in rotating machinery. Rolling bearing failure will directly cause mechanical equipment defects, resulting in serious economic losses and safety risks. Therefore, it is necessary to conduct in-depth research on rolling bearing fault diagnosis methods (Glowacz et al., 2018; Bin et al., 2018). The traditional fault diagnosis method still stays under constant speed condition, but in the actual working conditions, the rolling bearing mostly works under variable speed condition, which brings great challenges to the traditional fault diagnosis methods, and the fault diagnosis of rolling bearing under variable speed condition have become a hot issue in the current study (Gao et al., 2020; Li et al., 2020; Ma et al., 2020).

Under variable speed conditions, there are two major categories of fault diagnosis methods for rolling bearings. One is the order analysis method (Wang et al., 2020; Chen et al., 2019; Wu et al., 2019). The essence is to convert the variable-speed time-domain signal into stationary angle signal, and then apply the constant-speed fault diagnosis method to stationary angle signal to achieve fault diagnosis. The other is that the time-frequency analysis method is 
directly applied to the vibration signal to extract fault information (Liu et al., 2018; Bo et al., 2019; Liu et al., 2019). By mapping the time-domain signal to the time-frequency domain, the instantaneous frequency information of the signal can be obtained, and the fault can be diagnosed. For the first category, order analysis methods include hardware order analysis, computational order analysis, and order analysis based on instantaneous frequency estimation. Among them, hardware order analysis requires the installation of complicated hardware equipment, resulting in inconvenient installation and high cost. In many practical applications, it is limited; computational order analysis simplifies the installation of equipment to a certain extent, but it still needs to install the key phase device, which is difficult to use universally; order analysis based on instantaneous frequency estimation avoids the problem of installing hardware equipment. The rotational speed curve is estimated directly from the vibration signal, and the signal is re-sampled according to the estimated rotational speed information to realize the order analysis. In general, the fault diagnosis method of variable-speed rolling bearing based on order analysis is complicated, with many steps, and there are many influencing factors, such as the precision of instantaneous frequency estimation and angular resampling and so on. The processing precision of each step will have a great influence on the results. For the second category, time-frequency analysis method, time-frequency analysis is very suitable for dealing with non-stationary signals, making up for the lack of only time domain or frequency domain analysis. It can simultaneously obtain the changes of the signal in the time domain and frequency domain. In the variable speed condition, the fault signal of rolling bearing presents complex amplitude modulation and frequency modulation and time-varying characteristic. The time-frequency analysis method can well reveal the time-varying information of non-stationary signals. For fault diagnosis for non-stationary vibration signals of rolling bearings under variable speed conditions, time-frequency analysis is a simple, straightforward and effective solution.

Under varying speed conditions, the fault characteristic frequency of rolling bearing changes with the change of speed, and it is characterized by strong time-varying characteristics. Therefore, the traditional frequency domain demodulation method cannot extract time-varying fault characteristic frequency. Only the time-frequency analysis method can extract the time-varying characteristics. Besides, in variable speed conditions, the fault characteristics of rolling bearings are often severely disturbed and even submerged in strong noise. Therefore, in order to extract fault information from vibration signal, a time-frequency analysis method which has high time-frequency concentration and good anti-noise property is needed.

Scholars have done a lot of research and application of time-frequency analysis methods. Traditional time-frequency analysis methods include short-time Fourier transform (STFT) (Amstrup et al., 2004; Tao et al., 2020), wavelet transform (Chen et al., 2019; Qin et al., 2020), Wigner-Ville distribution (Claasm et al., 1980; Mustafa et al., 2020) and Hilbert-Huang transform (Zoltan et al., 2019; Hoseinzadeh et al., 2019). These methods are used to analyze the vibration signal of variable-speed rolling bearing, and some results are obtained respectively. But these methods have many limitations that cannot be ignored. The short-time Fourier transform and wavelet transform are all limited by the Heisenberg uncertainty principle, and the time-frequency concentration is difficult to meet the requirements. The Wigner-Ville distribution has high time-frequency concentration, but there is a serious cross-interference problem, not suitable for analysis of complex multi-component signals. Zou et al. (2001) has theoretically proved that there is no Wigner-Ville distribution without cross-interference and with high time-frequency concentration. The modal aliasing problem of Hilbert-Huang transform brings great inconvenience to its application.

Among the newly emerged time-frequency analysis methods in recent years, the synchrosqueezing transform (SST) proposed in 2011 by Ms. Daubechies et al. (2011), one of the founders of wavelet transform, is the most popular. The essence of SST is the combination of wavelet transform and time-frequency rearrangement. SST has attracted wide attention as soon as it was proposed, but it has poor anti-noise performance and is not suitable for processing fast-varying frequency and multi-component signals. Many scholars continue to improve it, and apply it to the field of mechanical fault diagnosis, such as: Li et al. (2012) proposed generalized synchrosqueezing transform and applied it to the gear fault diagnosis, solving the problem of energy diffusion and decreasing time-frequency concentration in the analysis of fast-varying frequency signal for SST. Feng et al. (2015) pointed the problem of frequency aliasing when processing multi-component signals for SST, and proposed an iterative demodulation analysis method. Iterative decomposition is performed first, then SST is performed on each component to improve the time-frequency resolution of multi-component signals, and applied to the planetary gearbox fault diagnosis; Wang et al. (2018) proposed a matching synchrosqueezing transform method for processing rapidly changing multi-component signals and applied to mechanical fault diagnosis. In 2015, in order to solve the problem of poor anti-noise property of SST, Daubechies et al. 
(2016) further proposed the concentration of frequency and time via a multitapered synchrosqueezed transform(ConceFT), which improves noise immunity to a certain degree, combining multitaper estimation ideas and averaging over random projections with synchrosqueezing. Feng et al. (2017) directly applied it to the fault diagnosis of rolling bearing under variable speed, and achieved certain results. However, the method is complicated in process and low in computational efficiency, and is not suitable for analyzing a large amount of experimental data, so the practical application is limited.

In order to overcome the shortcomings of existing time-frequency analysis methods, inspired by SST, Yu et al. (2017) proposed a novel time-frequency analysis method called synchroextracting transform (SET) in 2017, which greatly improved the anti-noise performance and time-frequency concentration of SST. SET can still ensure high time-frequency concentration at low signal-to-noise ratio (SNR), and its principle is clear and concise, and it is a parameter-less method that does not require complicated parameter settings. With low computational complexity and high efficiency, SET can be used for real-time analysis, which is an efficient time-frequency analysis method. Yu Gang has proved that the time-frequency concentration of the SET is very high, its time-frequency concentration is higher than the current 99\% time-frequency analysis method; at low SNR, it has good noise robustness; it can be used for weak signal detection to extract weak fault characteristics; it is capable of accurate instantaneous frequency estimation of complex time-varying signals. Yu (2016) applied it to time-frequency analysis of excavator vibration signals and instantaneous frequency estimation of bat signal. Chen et al. (2017) dealt with seismic signals making use of SET and realized hydrocarbon detection. SET has not been widely used at present. Considering many excellent merits of SET, it can be known that it is very suitable for analyzing non-stationary time-varying signals. For rolling bearing fault signal, SET can accurately extract the time-varying fault characteristic frequency, so it can provide an effective time-frequency demodulation analysis method for rolling bearing fault diagnosis under variable speed.The rolling bearing vibration signal contains a large amount of noise and interference in irrelevant frequency bands. In the traditional sense, band-pass filtering of the vibration signal to select the optimal frequency band for fault sensitivity is a necessary preprocessing step. The fast spectral kurtosis method (Borghesani et al., 2014) is a simple and effective method for selecting the optimal parameters of the band-pass filter.

The main contributions of this paper are as follows: (i) Combining Hilbert transform with SET, the envelope demodulation method based on SET for fault diagnosis of rolling bearings under variable speed is proposed. The amplitude envelope of the signal is calculated by the Hilbert transform and SET is applied to analyze the amplitude envelope. So the fault information can be extracted from the envelope demodulation spectrum. The proposed algorithm not only improves the time-frequency concentration of rolling bearing vibration signal under variable speed condition, but also improves the estimation accuracy of fault characteristic frequency. (ii) In order to make the algorithm have better applicability to the vibration signal with strong noise, the fast spectral kurtosis is used to design adaptive band-pass filter, and the signal is filtered before Hilbert transform to increase the noise robustness of the algorithm.

Hereafter, this paper is structured as follows. In Section 2, relevant principles including fast spectral kurtosis and SET are produced, and concrete steps of the proposed method are illustrated. In Section 3, a numerical simulated rolling bearing signal is analyzed to prove effectiveness and high time-frequency concentration of the proposed method compared with STFT and SST. In Section 4, the lab experimental vibration signals of rolling bearing with rising speed and fluctuate speed are employed to further validate the practicability of the proposed method. At last, the conclusions are given in Section 5.

\section{Theory}

\subsection{Fast spectral kurtosis}

\subsubsection{Spectral kurtosis}

Spectral kurtosis is an effective indicator in time-frequency domain describing impulse characteristics of signal. The stronger the signal impulse is, the larger the spectral kurtosis is. So the spectral kurtosis is often used as the indicator to select the most impulsive part of signal in frequency domain. Kurtogram is an accurate and straight representation of spectral kurtosis, in which the optimal center frequency and bandwidth can be obtained. Its general principle is as follows.

The Wold-Crammer decomposition of non-stationary signal $x(t)$ is defined as

$$
Y(t)=\int_{-\infty}^{+\infty} e^{j 2 \pi f t} H(t, f) d X(f)
$$


where $H(t, f)$ is complex envelope in frequency $f$ of $Y(t) . H(t, f)$ contains some information described by spectrum moment shown in Eq. (2).

$$
S_{2 n Y}(f)=E\left\{S_{2 n Y}(t, f)\right\}=E\left\{|H(t, f) d X(f)|^{2 n}\right\} / d f=E\left\{|H(t, f)|^{2 n}\right\} S_{2 n x}
$$

The forth-order spectral cumulant of $Y(t)$ is defined as

$$
C_{4 Y}(f)=S_{4 Y}(f)-2 S_{2 Y}^{2}(f), f \neq 0 .
$$

The final spectrum kurtosis of $Y(t)$ can be obtained by

$$
K_{Y}(f)=\frac{C_{4 Y}(f)}{S_{2 Y}^{2}(f)}=\frac{S_{4 Y}(f)}{S_{2 Y}^{2}(f)}-2, f \neq 0
$$

The fault features of rolling bearing usually show impulse characteristics. The fault features are hidden in the shock signal. The more obvious the shock is, the more fault features are included, and the greater the spectral kurtosis is.

\subsubsection{Fast spectral kurtosis}

The fast spectral kurtosis is an effective algorithm with high computational efficiency. In the paper, the fast kurtogram algorithm based on tree filter banks is used to adaptively determine the optimal center frequency and bandwidth of the band-pass filter. According to the selected parameters, the band-pass filter is constructed to filter the fault signal. The steps of the fast kurtogram algorithm are as follows:

(i). A low-pass filter $h_{0}(n)$ whose bandwidth is $[0,1 / 4]$ and a high-pass filter $h_{1}(n)$ whose bandwidth is $[1 / 4,1 / 2]$ are constructed respectively as:

$$
\begin{aligned}
& h_{0}(n)=h(n) e^{j \pi n / 4} \\
& h_{1}(n)=h(n) e^{j 3 \pi n / 4}
\end{aligned}
$$

where $h(n)$ is a low-pass filter with a cutoff frequency of $1 / 8$ (normalized frequency)。The signal is filtered by $h_{0}(n)$ and $h_{1}(n)$, then the sub-band signal is obtained by twice down sampling, as shown in Fig. 1. According to this method, the signal is decomposed and filtered layer by layer, so $2^{k}$ sub-band signals are obtained at the $k$ th level.

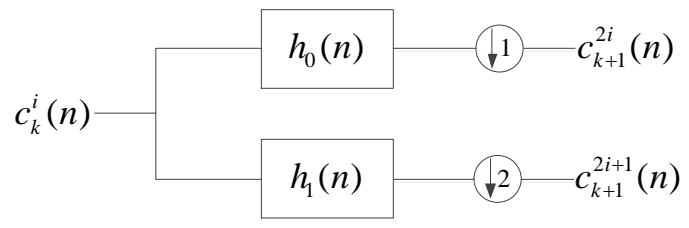

Fig. 1 Filtering and decomposition.

(ii). The coefficient $c_{k}^{i}(n), i=0 \cdots 2^{k}-1$ is obtained after the signal $x(k)$ passes through the $i$ th filter at the $k$ th level. $c_{k}^{i}(n)$ is the sub-signal obtained by down sampling the original signal after filtering by a narrow-band filter with center frequency $f_{i}=\left(i+2^{-1}\right) 2^{-k-1}$ and bandwidth $(\Delta f)_{k}=2^{-k-1}$. The frequency partition of sub -signals in each level is shown in Fig. 2. 


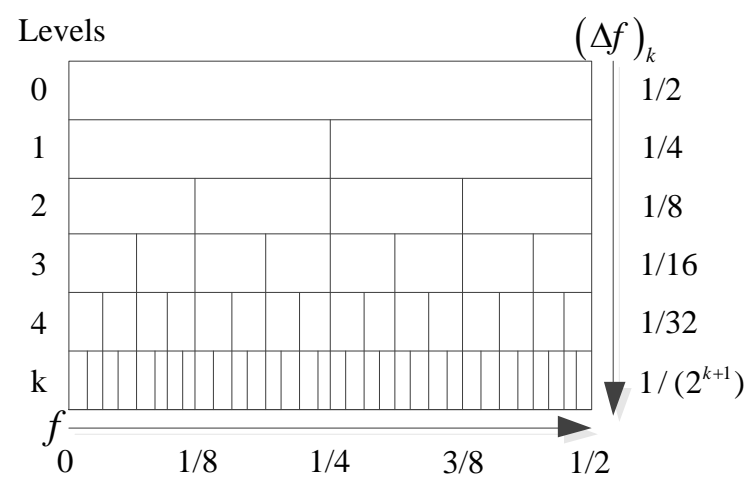

Fig. 2 Frequency and frequency resolution partition of sub-signals in each level.

(iii). According to Eq. (4), the spectral kurtosis of each sub-signal $c_{k}^{i}(n)$ at the central frequency $f_{i}=\left(i+2^{-1}\right) 2^{-k-1}$ and bandwidth $(\Delta f)_{k}=2^{-k-1}$ can be obtained. The results of spectral kurtosis are as follows:

$$
K=\frac{E\left\langle\left|c_{k}^{i}(n)\right|^{4}\right\rangle}{\left(E\left\langle\left|c_{k}^{i}(n)\right|^{2}\right\rangle\right)^{2}}-2
$$

All the spectral kurtosis are summed up to get the kurtosis values of the original signal under different band-pass filters in the whole frequency band, which is named fast kurtogram. In the fast kurtogram, the different color blocks represent the time-frequency information of different parts of the signal. The darker the color part, the greater the spectral kurtosis is and the stronger the impact component in the corresponding signal. This is the selected optimal frequency band. The original signal is band-pass filtered based on the resulting center frequency and bandwidth, and the signal containing the most fault information can be obtained.

\subsection{Synchroextracting transform}

SET is a new time-frequency analysis method, and it is a post-processing procedure of the STFT, which is a more energy concentrated time-frequency representation than classical time-frequency analysis methods and can effectively describe time-frequency characteristics.

For a multi-component signal $s(t)$, which can be seen as the sum of $n$ non-stationary modes, its expression is as follows

$$
\begin{aligned}
& s(t)=\sum_{k=1}^{n} s_{k}(t)=\sum_{k=1}^{n} A_{k}(t) e^{i \varphi_{k}(t)} \\
& \varphi_{k+1}^{\prime}-\varphi_{k}^{\prime}>2 \Delta
\end{aligned}
$$

where $s_{k}, A_{k}$ and $\varphi_{k}$ respectively denote the $k$ th mode, the corresponding instantaneous amplitude and instantaneous phase. $\varphi_{k}^{\prime}$ is the first-order derivative of $\varphi_{k}$, and denotes instantaneous frequency. $\Delta$ is the frequency support of window function.

SET is based on STFT, the STFT representation $G_{e}(t, \omega)$ of signal $s(t)$ is shown as following

$$
G_{e}(t, \omega) \approx \sum_{k=1}^{n} A_{k}(t) \hat{g}\left(\omega-\varphi_{k}^{\prime}(t)\right) e^{j \varphi_{k}(t)}
$$

where $\hat{g}()$ denotes the Fourier transform of the window function $g$.

According to Eq. (10), the instantaneous frequency can be calculated by 


$$
\varphi^{\prime}(t, \omega)=\sum_{k=1}^{n} \varphi_{k}^{\prime}(t, \omega)=-i \frac{\partial_{t} G_{e}(t, \omega)}{G_{e}(t, \omega)}
$$

In order to enhance the time-frequency concentration, Yu et al. (2017) designed an operator to only retain the time-frequency information most related to time-frequency characteristics of the target signal from STFT representation, which can remove the irrelevant interference and smeared time-frequency energy. The SET is formulated as

$$
T e(t, w)=G_{e}(t, \omega) \delta\left(w-\varphi^{\prime}(t, \omega)\right)
$$

where $\delta\left(\omega-\varphi^{\prime}(t, \omega)\right)=\left\{\begin{array}{l}1, \omega=\varphi^{\prime}(t, \omega) \\ 0, \text { else }\end{array}\right.$, which is named the synchroextracting operator (SEO).

According to Eq. (10) and Eq. (12), the following expression can be deduced.

$$
\left.T e(t, w)\right|_{\omega-\sum_{k=1}^{n} \varphi_{k}^{\prime}(t)=0}=\left.G_{e}(t, \omega)\right|_{\omega-\sum_{k=1}^{n} \varphi_{k}^{\prime}(t)=0} \approx \sum_{k=1}^{n} A_{k}(t) \hat{g}(0) e^{i \varphi_{k}(t)}
$$

In this way, we can obtain a sharper time-frequency representation than STFT and extract instantaneous frequency with a highly precise degree.

\subsection{Analysis procedure}

Rolling bearing faults behave impulse characteristics in vibration signals, and obvious impulse features usually are contained in the resonance band. However, in practice, fault impulse features usually are hidden by strong noise. Thus, it is necessary to separate the optimal frequency band containing the most fault impulse features from irrelevant interference. In this paper, fast spectral kurtosis is adopted to select the optimal frequency band to realize adaptive band-pass filtering.

There is one problem that cannot be ignored. The filtered signal also contains a lot of noise, which still interfere with the analysis results. Furthermore, rolling bearing vibration signals show strong non-stationary behavior at variable speed. Fault features are often submerged in noise and the nonstationarity is difficult to extract. So a well time-frequency analysis method with good anti-noise property and time-frequency concentration is necessary. SET possesses the above motioned excellent properties, which is an appropriate time-frequency analysis method to extract the time-varying fault features from non-stationary signal with noise.

For rolling bearing vibration signal, fault features are basically contained in its amplitude envelope signal. Moreover, amplitude envelope signal is free from carrier signal resonance interference, and amplitude envelope demodulation spectrum is basically not effected by complex frequency sidebands around resonance frequency. Thus, fault characteristic frequency can be extracted clearly in time-frequency representation of amplitude envelope signal. Therefore, the envelope demodulation method based on SET for fault diagnosis of rolling bearing under variable speed is proposed.

For an actual rolling bearing vibration signal, the proposed method can be generated following the procedure listed below:

(i). Fast spectral kurtosis. Perform fast spectral kurtosis on rolling bearing vibration signal. On fast kurtogram, the optimal frequency band can be got, and center frequency and band width are all displayed in the kurtogram.

(ii). Band-pass filtering. Design band-pass filter according to parameters obtained by fast spectral kurtosis. Obtain the optimal part of original signal containing the most fault impulse.

(iii). Amplitude envelope. Perform Hilbert transform on the band-pass filtered signal to obtain the amplitude envelope signal.

(iv). Time-frequency demodulation. Perform SET on the amplitude envelope signal to get amplitude envelope signal's time-frequency demodulation spectrum. Fault characteristic frequency can be represented in the time-frequency demodulation spectrum.

The numerical validation and actual signal verification of the proposed method are respectively performed below. 
The advantages of the proposed method are highlighted by comparison with STFT and SST. In the simulation signal, two tests are done, one is the anti-noise performance test, and the trend of time-frequency concentration variation with SNR is summarized. The other is the robust performance test, which analyzes the effectiveness of the proposed method under failure conditions of the system itself (i.e. failure of the adaptive band-pass filter in this paper). At last, actual rolling bearing vibration signals respectively under rising speed and fluctuated speed are used to verify the effectiveness and practicability of the proposed method.

\section{Numerical validation}

\subsection{Signal design}

To validate the performance of the proposed method, a numerical simulated rolling bearing vibration signal with a local outer race fault under variable speed condition is designed. The simulation signal is as follows

$$
x(t)=(-0.15 t+2.1) \times \sum_{m=1}^{N} \exp \left(-b\left(t-t_{m}\right)\right) \sin \left(2 \pi f_{0}\left(t-t_{m}\right)\right) u\left(t-t_{m}\right)+n(t)
$$

where $n(t)$ denotes Gaussian white noise, $u(t)$ is unit step function, $b$ is structural attenuation coefficient, $f_{0}$ is resonance frequency due to bearing fault, and $t_{m}$ represents the time when the $m$ th impact occurred, which can be deduced by

$$
\left\{\begin{array}{l}
t_{1}=(1, N+u) \times 1 / f_{r}\left(t_{0}\right) / k \\
t_{m}=(1+u) \times 1 / f_{r}\left(t_{m-1}\right) / k \quad m=2,3, \cdots, N
\end{array}\right.
$$

where $t_{0}=0, u$ is error coefficient, $f_{r}(t)$ denotes bearing rotation frequency and $k$ is fault feature coefficient.

In the paper, the parameters of the simulated signal are shown in Table 1.

Table 1 The parameters of the simulated signal.

\begin{tabular}{c|c|c|c|c|c|c}
\hline \hline SNR & $b$ & $f_{0}$ & $k$ & $u$ & sampling frequency & $f_{r}(t)$ \\
\hline $0 \mathrm{~dB}$ & 100 & $3200 \mathrm{~Hz}$ & 3.7 & 0.01 & $24000 \mathrm{~Hz}$ & $10 \times\left(15-0.1 e^{1.2 t}\right) / 15$ \\
\hline
\end{tabular}

According to bearing rotation frequency and fault feature coefficient, the fault characteristic frequency $f_{c}=3.7 f_{r}$. The next task is to extract the fault characteristic frequency and its frequency multiplier to diagnose bearing failures.

\subsection{Envelope demodulation analysis and comparison analysis}

The time domain waveform and Fourier spectrum of the simulation signal are separately shown in Figs. 1(a) and (b). We can see that there is a lot of noise in the signal. From Fourier spectrum we can see that distinct impact occurs near resonance frequency $3200 \mathrm{~Hz}$, and time-varying sideband appears around in the region of 2000-4000Hz. However, because of complex time-varying sidebands characteristics due to variable speed and noise interference, the fault information cannot be directly obtained from the Fourier spectrum.

Therefore, in order to extract fault features, we need to select the optimal frequency band for time-frequency analysis. First, determine the optimal frequency band according to fast spectral kurtosis. The fast kurtogram is shown in Fig. 3(c). It can be seen from the Fig. 3(c) that the maximum kurtosis value which is 51.6 is obtained at the optimal frequency band with center frequency $f=2976.56 \mathrm{~Hz}$ and bandwidth $B_{w}=2000 \mathrm{~Hz}$. It means that if the signal is band-pass filtered based on center frequency $f=2976.56 \mathrm{~Hz}$ and bandwidth $B_{w}=2000 \mathrm{~Hz}$, the filtered signal will contain the most fault information. Then, perform adaptive band-pass filtering on the signal according to the resulting filter parameters, and then calculate the amplitude envelope of the filtered signal via Hilbert transform. The amplitude envelope is shown in Fig. 3(d). Finally, the envelope signal is analyzed by SET, the SET time-frequency representation is shown in Fig. 3(e). In order to make the results clearer, we intercepted part of the time axis and frequency axis, and the Fig. 3(f) shows the comparison of theoretical fault characteristic frequency (FCF) curve and demodulated fault characteristic frequency by SET. It can be seen from the Figs. 3(e) and (f) that the time-varying non-stationarity caused by variable speed and strong noise interference show little influence on the result. The fault characteristic frequency 
and its multiplying frequency are clearly demodulated and perfectly coincide with the theoretical fault characteristic frequency curve, which can accurately determine bearing fault.

To compare and highlight the advantages of this method, the time-frequency demodulation analysis of the envelope signal is performed using STFT and SST, respectively. The results are shown in Figs. 1(g) and (h) and (i). It can be seen that although they can also roughly demodulate the fault characteristic frequency, they are more severely affected by noise and have energy divergence on different levels. STFT has poor time-frequency concentration, the result smears severely, and noise interference is serious, so it cannot meet requirements. The time-frequency concentration of SST is improved compared with STFT. But from Fig. 3(g), we can see that there exists edge effect, and the energy at the initial position is divergent severely, which will have a great impact on the extraction of fault characteristic frequency. Through the above comparative analysis, we can see that the proposed method has obvious advantages over other methods in anti-noise and time-frequency concentration, and it is an effective fault diagnosis method.

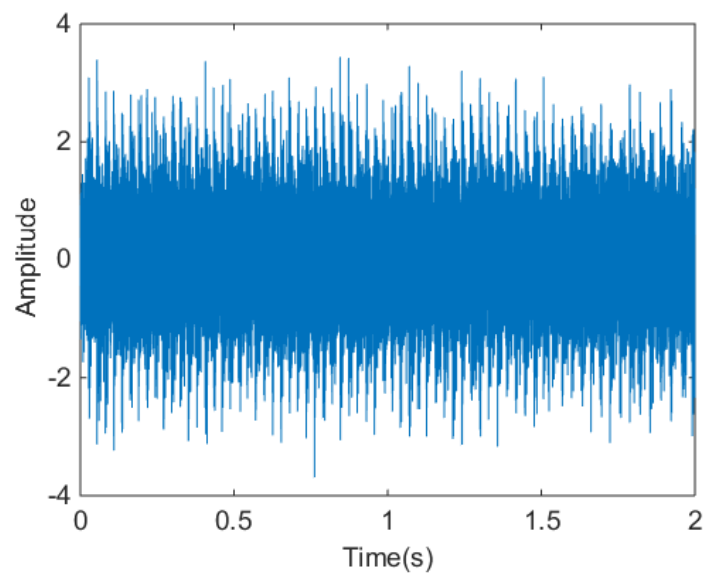

(a) The time domain diagram

fb-kurt.2 - $\mathrm{K}_{\max }=51.6 @$ level 1.5, Bw= 2000 Hz, $\mathrm{f}_{\mathrm{c}}=2976.5625 \mathrm{~Hz}$

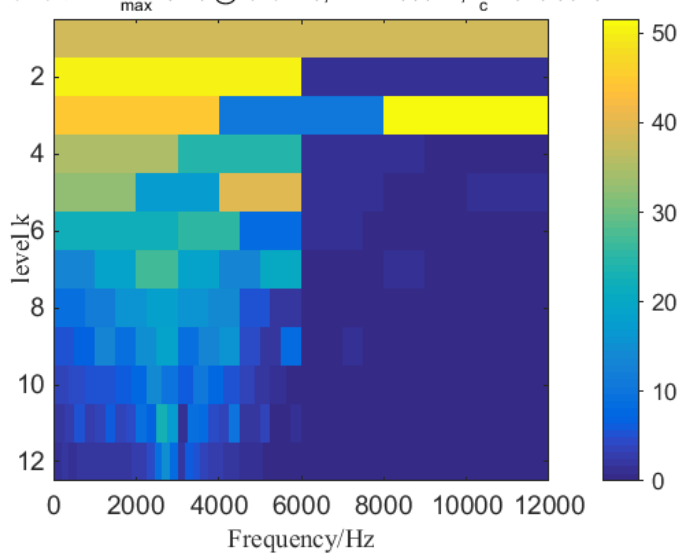

(c) The kurtogram

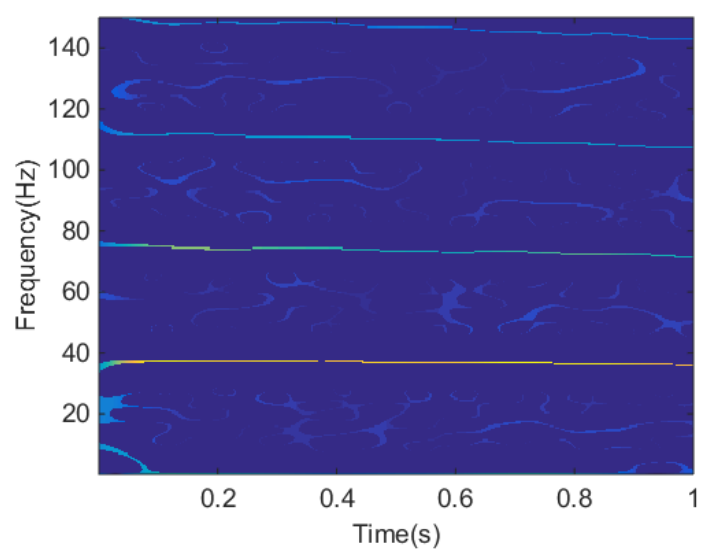

(e) SET

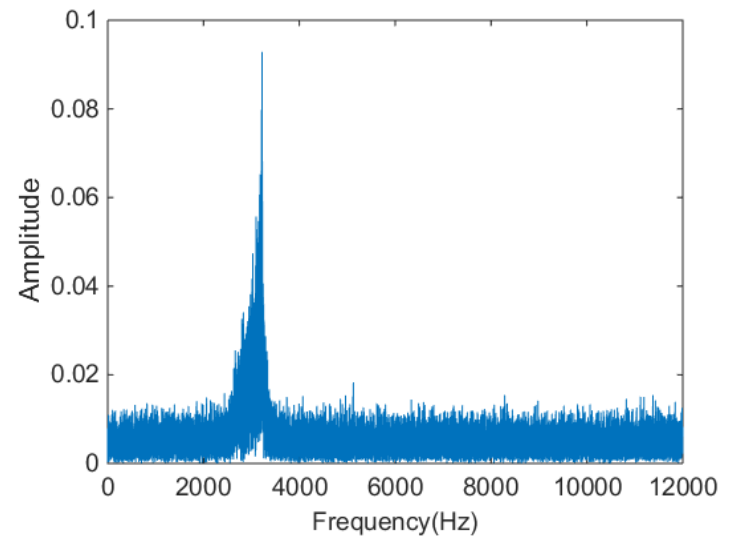

(b) The frequency domain waveform

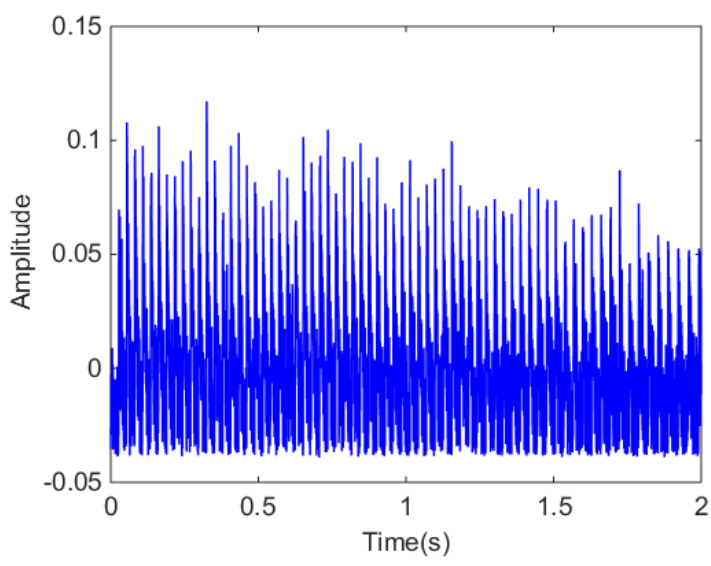

(d) The amplitude envelope

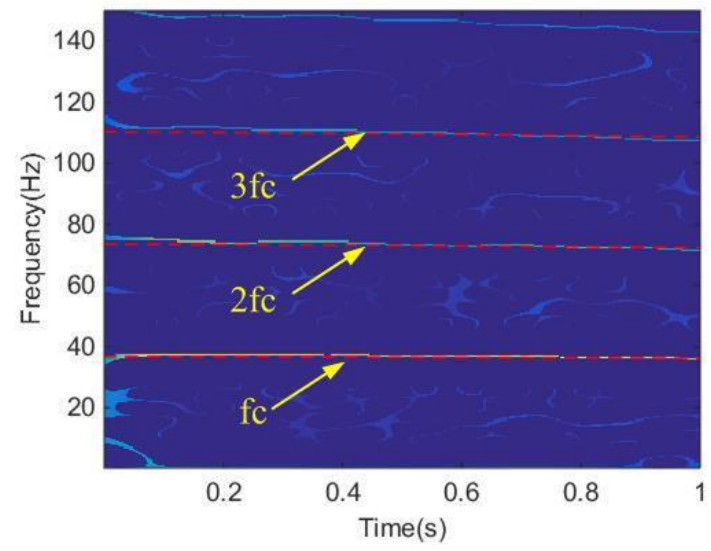

(f) Comparison of SET and real result 


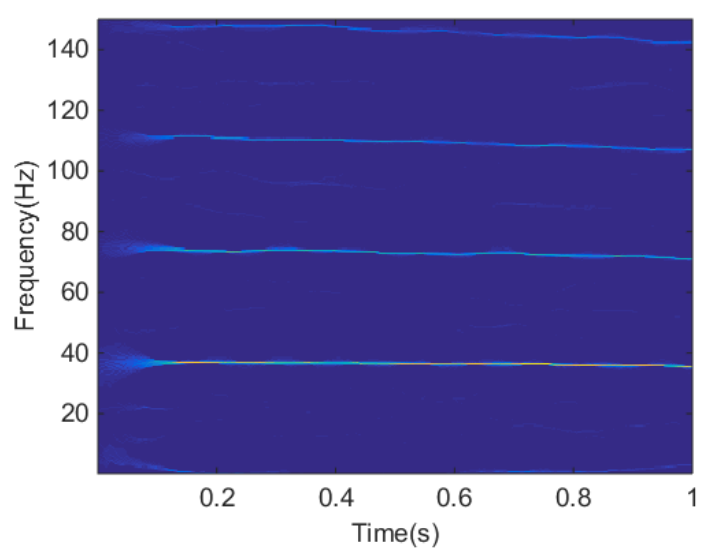

(g) SST

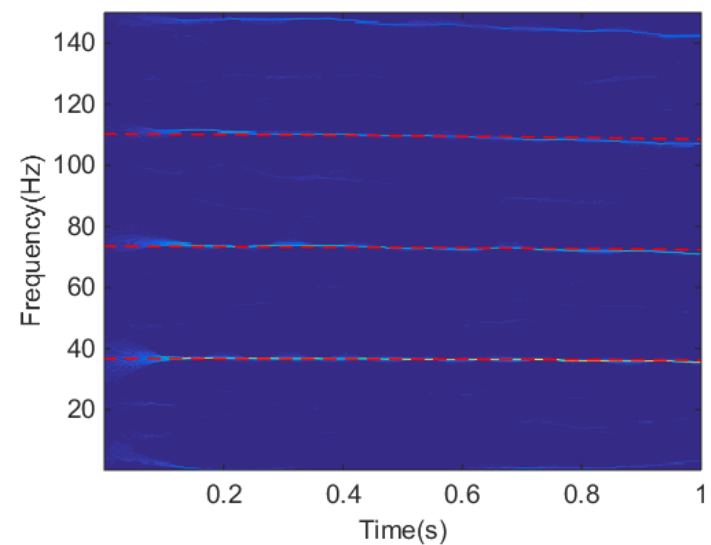

(h) Comparison of SST and real result

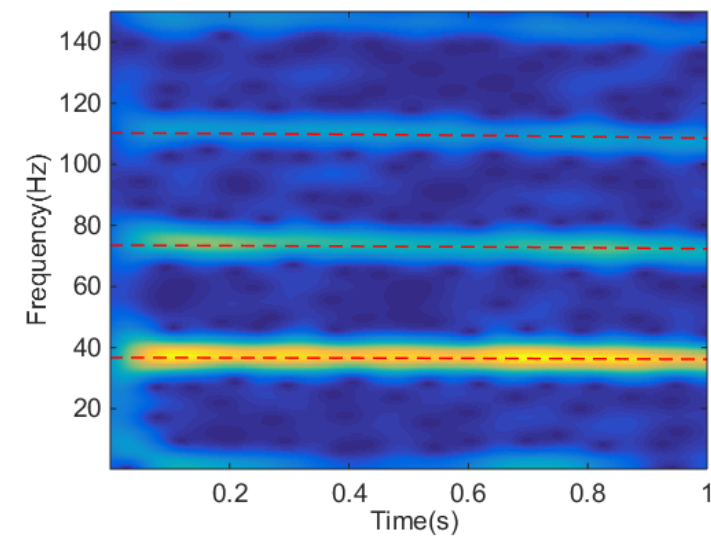

(i) Comparison of STFT and real result

Fig. 3 Simulation signal analysis results.

\subsection{Anti-noise performance and time-frequency concentration analysis}

The pros and cons of time-frequency concentration are important factors for evaluating the time-frequency analysis method. In order to quantify the advantages of the proposed method, The Rényi entropy is used as an index to measure time-frequency concentration. The smaller the Rényi entropy is, the higher the time-frequency concentration is. The relationship between Rényi entropy and SNR is studied to prove that this method has strong anti-noise and high time-frequency concentration. Calculate the Rényi entropy of the three methods under different SNR, as shown in Fig. 4. It can be seen that the overall time-frequency concentration of STFT is the worst, followed by SST, and the time-frequency concentration of SET is the best. From the point of view of noise robustness, the Rényi entropy of SET does not fluctuate when the SNR changes, and it always remains at a high level. This result once again strongly proves the advantages of the proposed method.

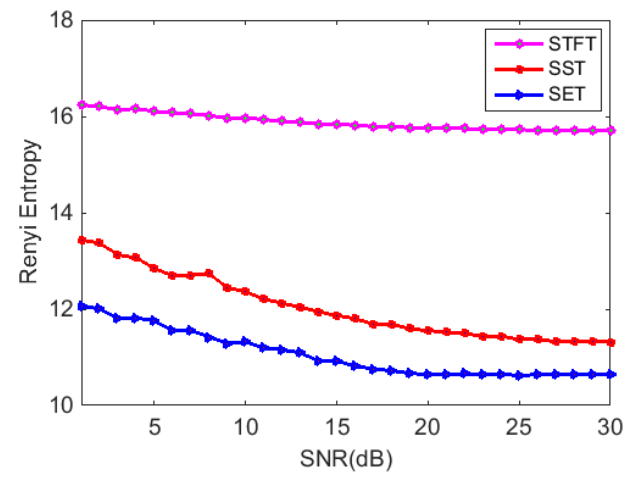

Fig. 4 The Rényi entropy of three methods under different SNR. 


\subsection{Bearing fault diagnosis robust performance test with band-pass filter failure}

Band-pass filter failure is a common sudden failure. In many cases, strong noise interference will cause the adaptive band-pass filter based on spectral kurtosis to fail. The performance of failure is only a rough determination of the center frequency, but the quality factor is very low, that is, the pass band of the band-pass filter is wider, even the full frequency band. Therefore, the failure of the band-pass filter will bring a lot of noise interference to the following fault characteristic frequency extraction. The sudden failure will have great influence on the analysis. In this case, we test the proposed method. If it is still valid, it shows that the proposed method has good robust performance, strong anti-interference ability and self-adaptive ability. The result is shown in Fig. 5. It can be seen from Fig. 5 that in the case of failure of the band-pass filter, the proposed method can still suppress noise interference and clearly extract the fault characteristic frequency, which is still consistent with the theoretical fault characteristic frequency. The result proves that the proposed method has good robustness and anti-interference property, and it still works well when filter fails suddenly.

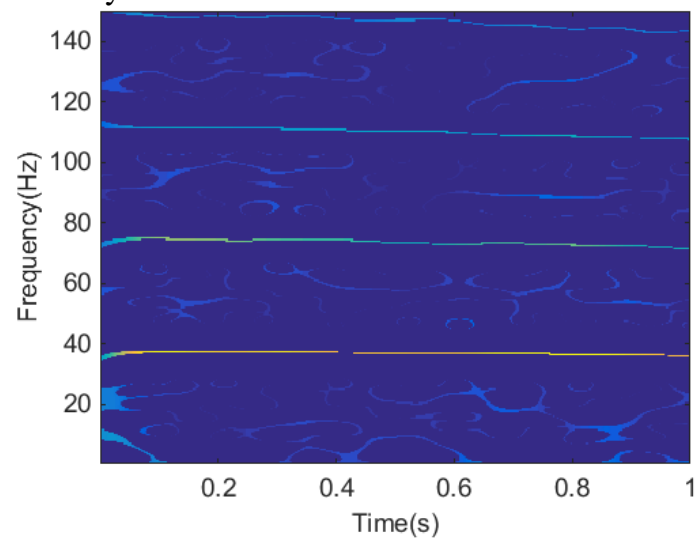

(a) SET with filter failure

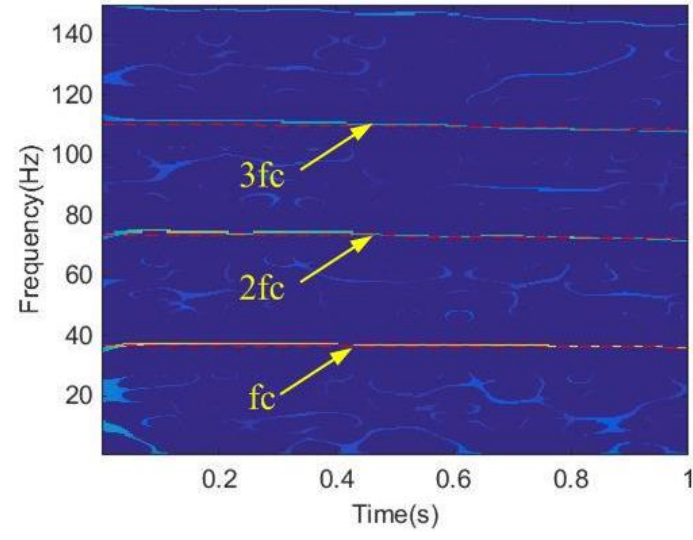

(b) Comparison of SET with filter failure and real result

Fig. 5 The result of proposed method under the failure condition of band-pass filter.

\section{Experimental tests of rolling bearing fault vibration signal \\ 4.1. Experimental settings}

In this section, we apply the proposed method to the real rolling bearing fault vibration signal to further validate the practicability and effectiveness. The real data is from the mechanical fault diagnosis laboratory and the simulation experiment platform and test rolling bearing are displayed in Fig. 6. As shown in Fig.6 (a), QPZZ-II fault simulation platform is composed of seven main parts: drive motor, coupling, rotating disc, tachometer, load, bearing and acceleration sensor. The tachometer is XB40-I type. The bearing type is N205EM, the front end is normal bearing, and the back end is fault bearing to be tested. The outer race of the cylindrical roller bearing N205EM has no ribs and its inner race and outer race can move axially, which can adapt to the change of relative position between shaft and shell caused by thermal expansion or installation error. The bearing can accept large radial load and high limit speed, but can't accept axial load. In the simulation test, the bearing is used as a free end and the processing fault can be set in the outer race. The acceleration sensor is CA-YD-188 type whose frequency response range is $[0.3 \mathrm{~Hz} 2500 \mathrm{~Hz}] \mathrm{and}$ it is installed in the vertical position of the bearing box. And the drive motor is YVF801-4, which is AC variable frequency motor with speed range of 75-1450rpm. The load type is CZ-0.5 which is the magnetic powder brake. In the simulation, the load is 1 horsepower. The cleanliness and lubrication condition have great influence on bearing vibration signal. Therefore, clean the bearing carefully with clean gasoline or kerosene, and then apply clean and high-quality lubricating grease before the bearing can be installed and used. In the test, the bearing is kept in good lubrication condition, so the vibration change caused by poor lubrication is ignored. The test rolling bearing which is N205EM type with slight crack in the outer race is shown in Figs. 6(c) and (d). Moreover, the main parameters of the bearing are shown in Table 2. As shown in Fig. 6 (d), the bearing with outer race fault is simulated by using electrical discharge machining (EDM) technology to destroy the bearing. The EDM technology is a kind of processing method that produces high temperature by pulse discharge between electrode wire and workpiece to make the metal melt. To simulate different degrees of fault, different sizes of faults are made. For the outer race fault, The fault sizes of $4 \mathrm{~mm} \times 0.7 \mathrm{~mm} \times 1.1 \mathrm{~mm}, 4 \mathrm{~mm} \times 0.5 \mathrm{~mm} \times 0.5 \mathrm{~mm}$ and $4 \mathrm{~mm} \times 0.3 \mathrm{~mm} \times 0.3 \mathrm{~mm}$ with length, width and depth are used to simulate severe fault, moderate fault and weak fault respectively. Figure 6 (d) shows the 
moderate failure of the outer race, which is also used in the simulation below.
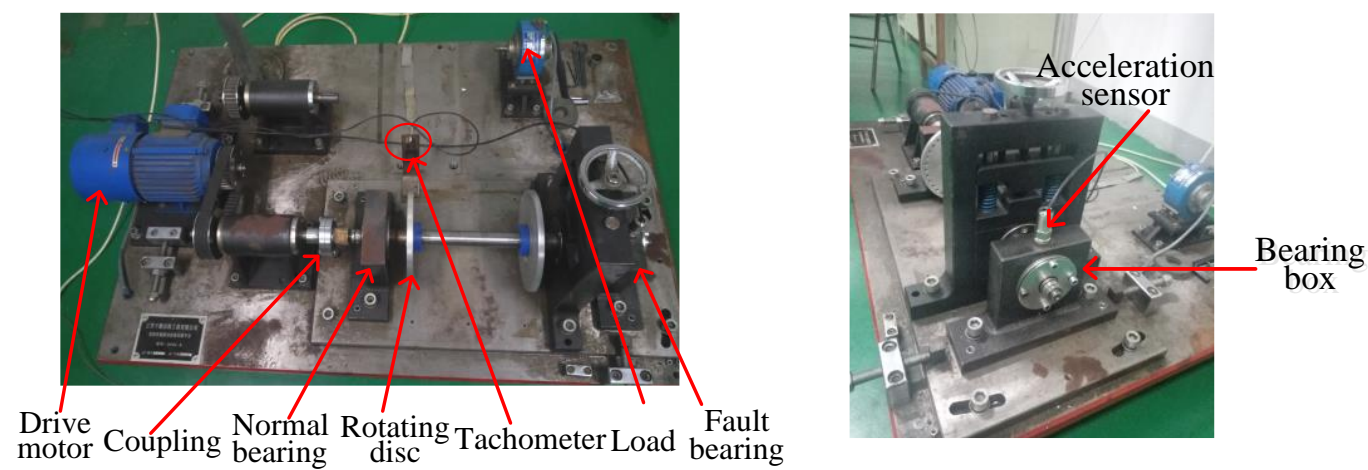

(a) QPZZ- II fault simulation platform

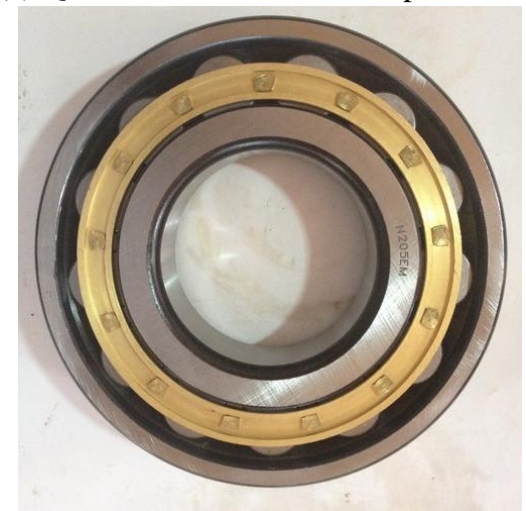

(b) The sensor installation position

(c) The test rolling bearing

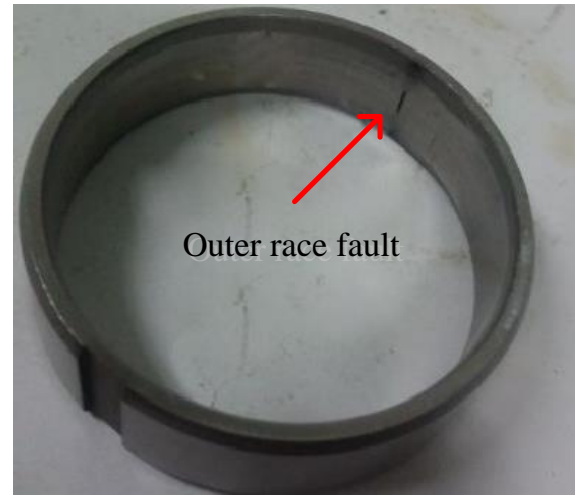

Fig. 6 QPZZ- II fault simulation platform and the test rolling bearing.

Table 2 The main parameters of the rolling bearing

\begin{tabular}{c|c|c|c}
\hline $\begin{array}{c}\text { Diameter of } \\
\text { pitch circle }\end{array}$ & $\begin{array}{c}\text { Diameter of } \\
\text { rolling body }\end{array}$ & $\begin{array}{c}\text { Contact } \\
\text { angle }\end{array}$ & $\begin{array}{c}\text { The number of } \\
\text { rolling bodies }\end{array}$ \\
\hline $38.5 \mathrm{~mm}$ & $7.5 \mathrm{~mm}$ & $0^{\circ}$ & 13 \\
\hline
\end{tabular}

In general, it is assumed that the outer race of the rolling bearing is fixed on the bearing seat, and only the inner race rotates with the shaft. The following assumptions are made:

(i). There is no sliding contact between rolling element and raceway.

(ii). Each rolling element has the same diameter and is evenly distributed between the inner and outer raceways.

(iii). There is no deformation of each part under radial or axial load.

So the outer race fault characteristic frequency formula is as follows

$$
f_{c}=f_{r} \cdot \frac{n}{2} \cdot\left(1-\frac{d}{D} \cos \alpha\right)
$$

where $f_{c}$ is fault characteristic frequency, $f_{r}$ is rotational frequency, $n$ denotes the number of rolling bodies, $d$ is diameter of rolling body, $D$ is diameter of pitch circle and $\alpha$ is contact angle.

The sampling frequency of vibration signal is $25600 \mathrm{~Hz}$. In order to well demonstrate the effectiveness of the proposed method, the analysis is carried out in the case of rising speed and complex fluctuated speed.

\subsection{Fault detection in rising speed condition}

The vibration signal waveform is shown in Fig. 7(a) which is rising speed condition with strong noise. And the simulation test is carried out for the moderate failure of the outer race whose fault size is $4 \mathrm{~mm} \times 0.5 \mathrm{~mm} \times 0.5 \mathrm{~mm}$. The 
rotational frequency varies from $11.4 \mathrm{~Hz}$ to $24.6 \mathrm{~Hz}$. According to Eq. (16), the fault characteristic frequency bandwidth of vibration is from $59.6676 \mathrm{~Hz}$ to $128.7564 \mathrm{~Hz}$. The Fourier spectrum is shown in Fig. 7(b). It can be seen that the frequency bands where the impactive component are located roughly in the area centered on 3000 and 9000 . It cannot be judged which part the fault information is concentrated in, so the filter parameter can be selected by fast spectral kurtosis filtering, the fast kurtogram is shown in Fig. 7(c). Similar to the analysis in Fig. 3 (c), the center frequency $f_{c}$ and bandwidth $B_{w}$ of the optimal band-pass filter are $3175 \mathrm{~Hz}$ and $800 \mathrm{~Hz}$ respectively. Then, the filtered signal is enveloped, as shown in Fig. 7(d). At last, perform SET on amplitude envelope, the result is shown in Fig. 7 (e). It can be seen that there are three prominent time-varying frequency curves in the graph. According to the bearing parameters and speed information, it can be determined that these frequency curves are consistent with the fault characteristic frequency curve and its multiplying frequency, from which it can be judged that the bearing is faulty. Figures 7(f) and (g) are the analysis results of SST and STFT, respectively. Compare the three methods, it can be seen that the fault frequency curve demodulated by the SST is rather fuzzy, the energy is divergent seriously, and the multiplying fault characteristic frequency cannot be extracted. Although STFT can clearly extract the fault characteristic frequency and multiplying frequency, the time-frequency concentration is poor, and there are too many redundant and irrelevant frequency interference. The proposed method has high time-frequency concentration, very strong ability to suppress noise, basically eliminates irrelevant frequency interference, and clearly demodulates single, double, and triple fault feature frequencies. Figure 7(h) shows the result of SET with filter failure, it can be seen that it is basically consistent with the result by fast kurtosis filtering, and can still accurately extract fault characteristic frequency. It can be proved that this method has stronger robustness and self-adaptive ability.

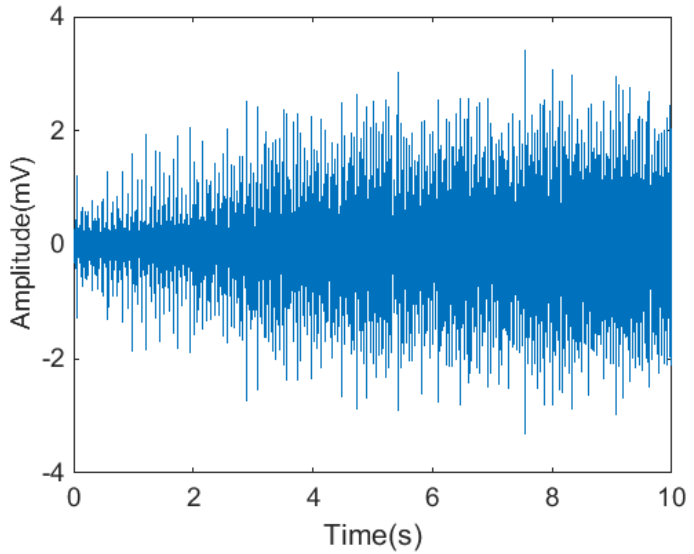

(a) The time domain diagram

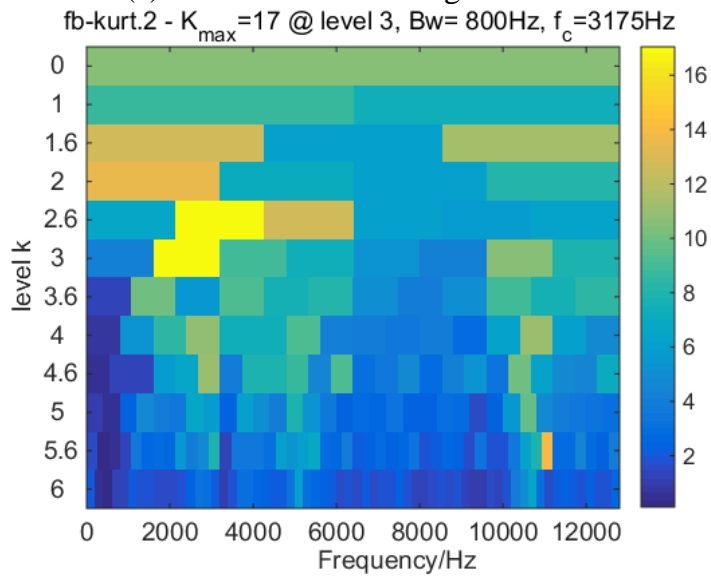

(c) The kurtogram

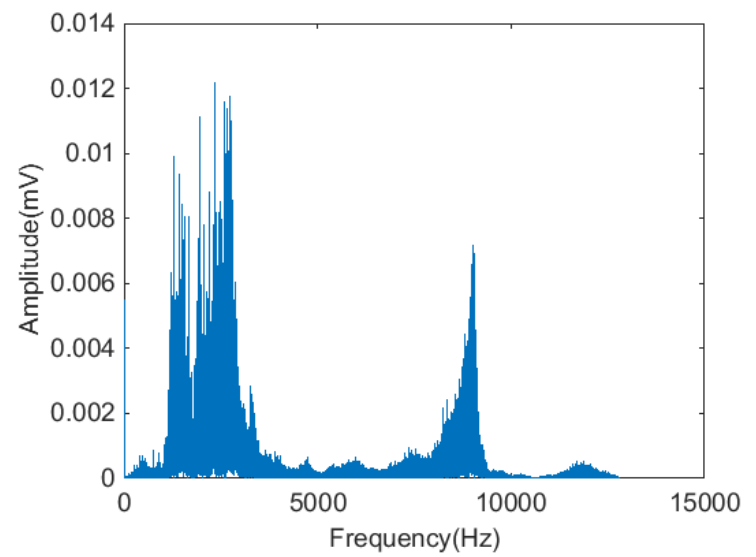

(b) The frequency domain waveform

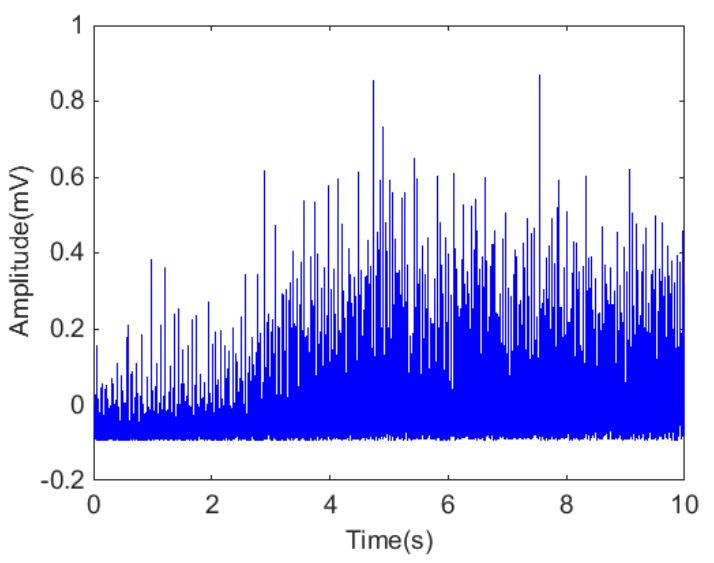

(d) The amplitude envelope 


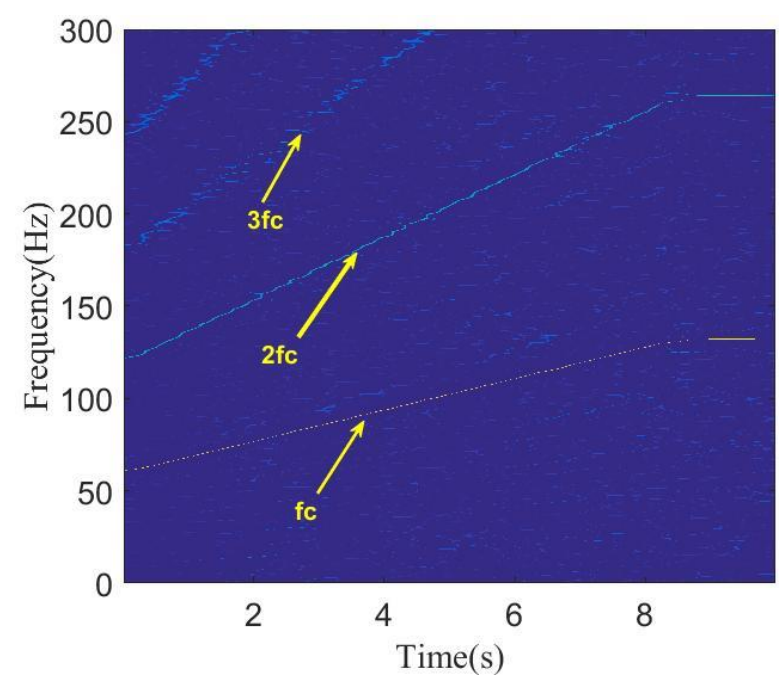

(e) SET

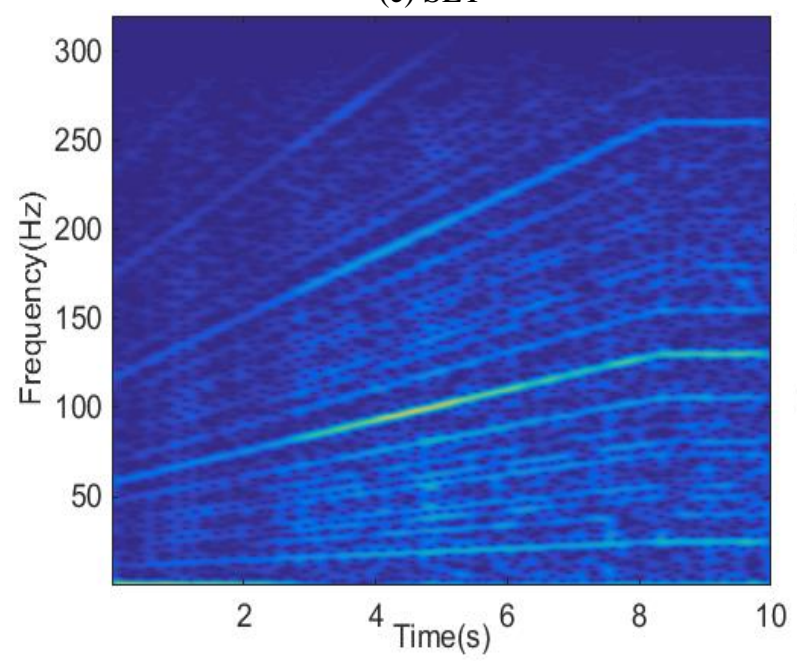

(g) STFT

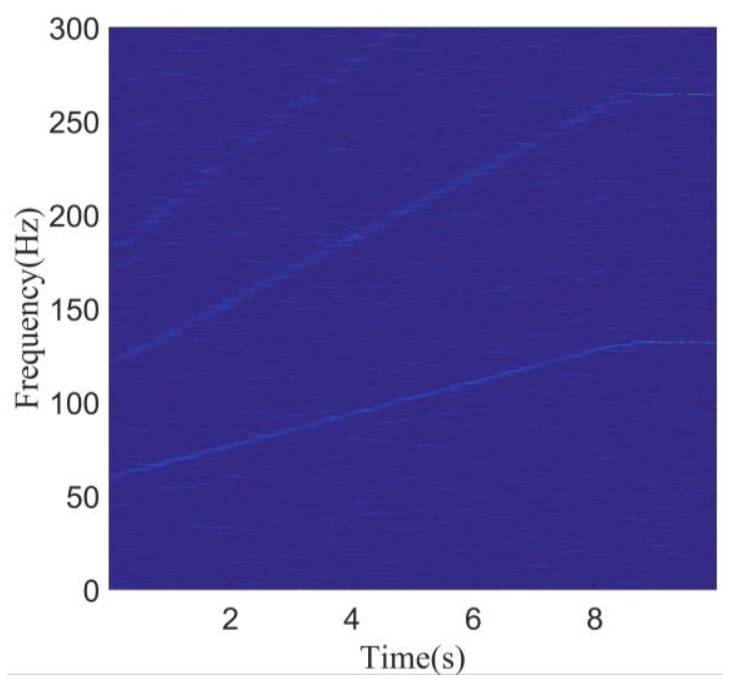

(f) SST

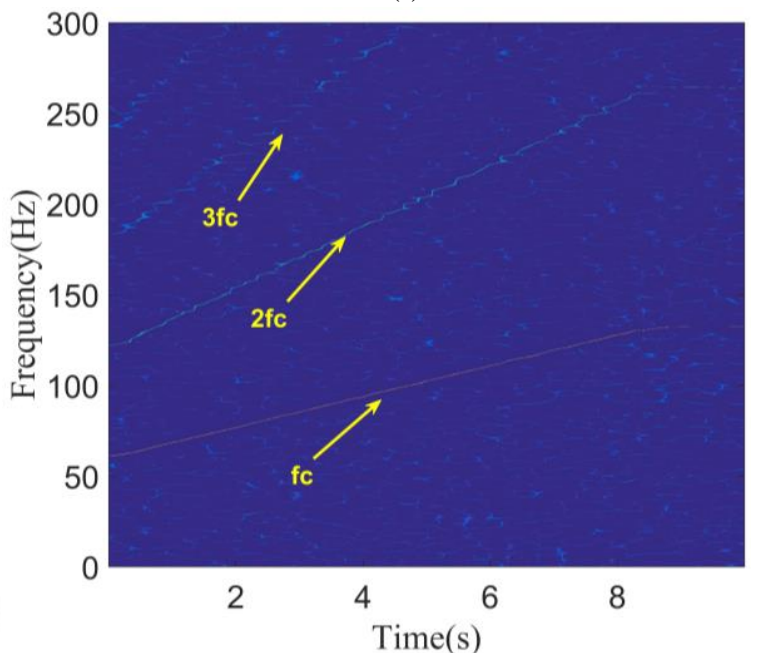

(h) SET with filter failure

Fig. 7 Real signal analysis results under rising speed.

In order to illustrate the range of fault sizes to which the method can be effectively applied, the simulation tests are carried out for the severe fault whose fault size is $4 m m \times 0.7 m m \times 1.1 \mathrm{~mm}$ and for weak fault whose fault size is $4 m m \times 0.3 \mathrm{~mm} \times 0.3 \mathrm{~mm}$. At the same time, to increase the practicability of the proposed algorithm, a simulation test is carried out for the outer race fault bearing with actual fault and the size of the minimum enclosing rectangle of actual fault is $5.32 \mathrm{~mm} \times 0.63 \mathrm{~mm} \times 0.3 \mathrm{~mm}$. So the outer race wear volume of actual fault is $1.0055 \mathrm{~mm}^{3}$ which is close to that of moderate fault and the wear volume of moderate fault is $1.0 \mathrm{~mm}^{3}$. The bearing with actual fault is shown in Fig.8.

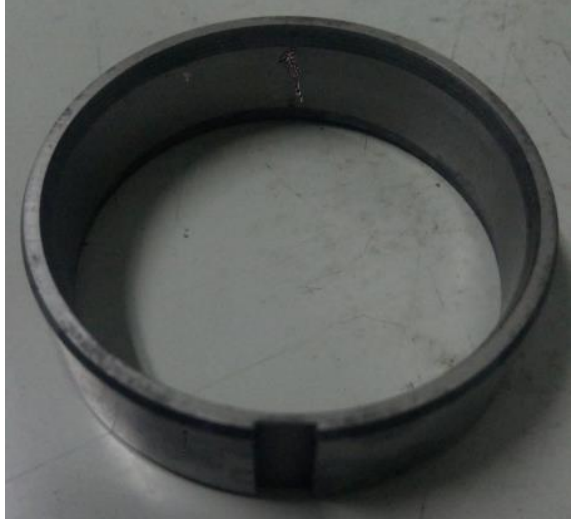

(a) The outer race with actual fault

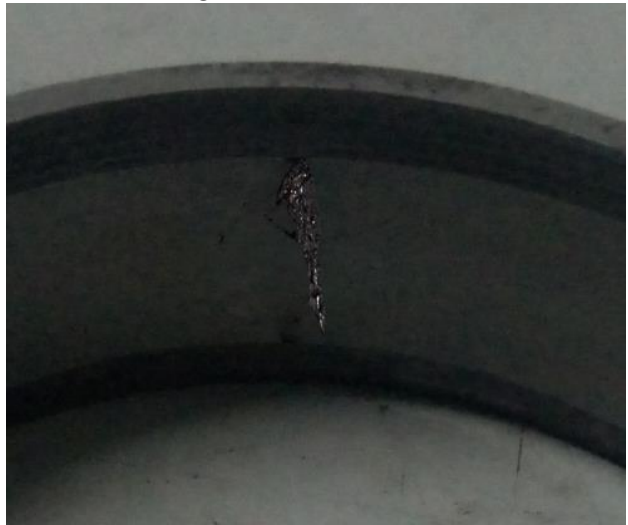

(b) The local enlarged drawing of the actual fault

Fig. 8 The outer race with actual fault. 
To quantitatively illustrate the effectiveness of the proposed algorithm, in addition to using the Rényi entropy mentioned in Section 3.3 to characterize the time-frequency concentration of the algorithm, the estimation error of the fault characteristic frequency as a precision evaluation index is introduced here. The real fault characteristic frequency is $f(t)$, and the estimate is $\hat{f}(t)$. The mean absolute error between them is used to represent the estimation error of the fault characteristic frequency, and its expression is as follows:

$$
\sigma=\frac{1}{m} \sum_{i=1}^{m}|\widehat{f}(i)-f(i)|
$$

where $m$ is the number of samplings.

The peak search algorithm is used to extract the estimation of the fault characteristic frequency in Figs. 7 (e), (f), (g) and (h), and the results are shown in Fig. 9 (a). Similarly, the fault characteristic frequency estimation curves under severe fault, weak fault and actual fault are obtained as shown in Figs. 9 (b), (c) and (d). The fault characteristic frequency curve of the actual fault is close to the theoretical characteristic curve of the outer race fault, so the actual fault can be judged as the outer race fault, and the diagnosis result is consistent with the actual fault location. The quantitative comparison results of different fault sizes are shown in Table 3.

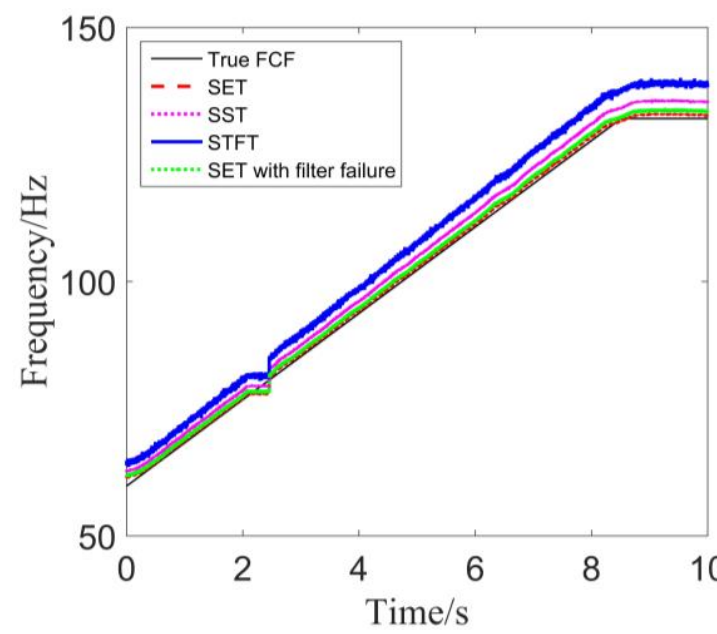

(a) Severe fault

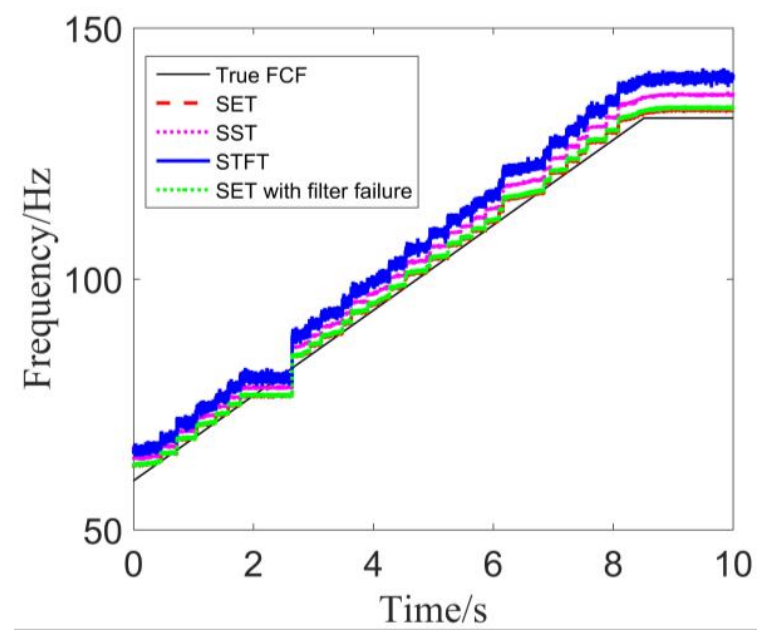

(c) Weak fault

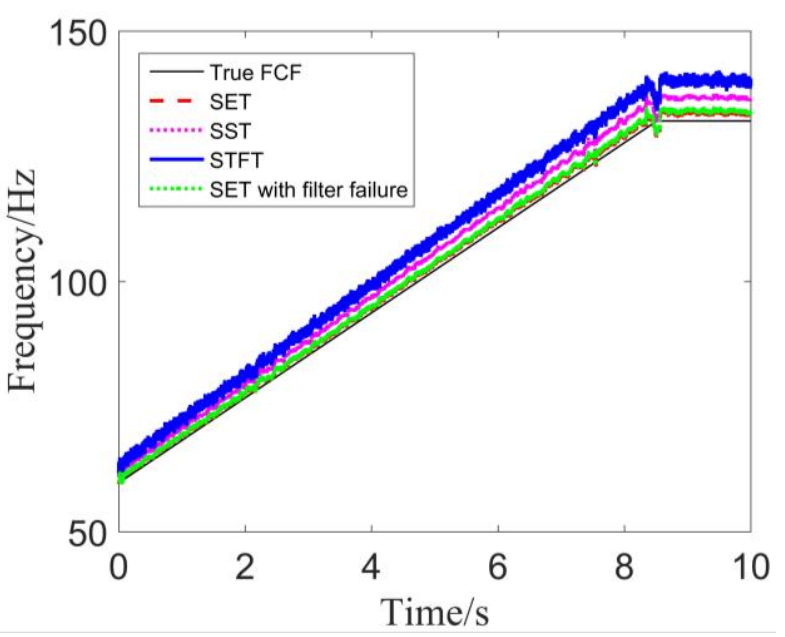

(b) Moderate fault

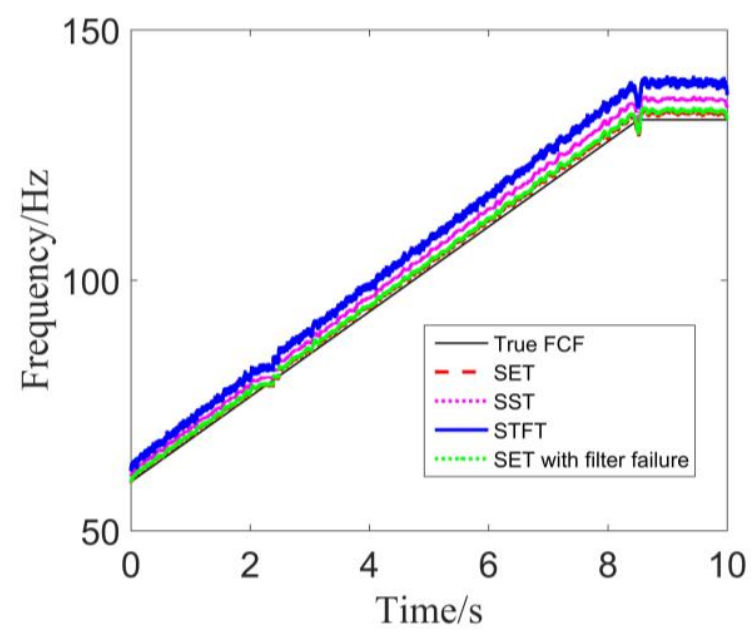

(d) Actual fault

Fig. 9 The estimation of fault characteristic frequency. 
Table 3 The comparison results of different fault sizes

\begin{tabular}{|c|c|c|c|c|c|}
\hline \multicolumn{2}{|c|}{ Pegree of fault } & Severe fault & Moderate fault & Weak fault & Actual fault \\
\hline \multirow{2}{*}{ SET } & $\begin{array}{l}\text { Rényi } \\
\text { entropy }\end{array}$ & 12.054 & 12.845 & 13.412 & 12.781 \\
\hline & $\begin{array}{l}\text { Estimation } \\
\text { error }(\mathrm{Hz})\end{array}$ & 0.547 & 0.625 & 1.548 & 0.588 \\
\hline \multirow{2}{*}{ SST } & $\begin{array}{c}\text { Rényi } \\
\text { entropy }\end{array}$ & 14.251 & 14.945 & 15.415 & 14.847 \\
\hline & $\begin{array}{l}\text { Estimation } \\
\text { error }(\mathrm{Hz})\end{array}$ & 2.154 & 2.569 & 4.547 & 2.341 \\
\hline \multirow{2}{*}{ STFT } & $\begin{array}{c}\text { Rényi } \\
\text { entropy }\end{array}$ & 16.214 & 17.022 & 18.451 & 16.812 \\
\hline & $\begin{array}{l}\text { Estimation } \\
\text { error(Hz) }\end{array}$ & 4.745 & 5.186 & 7.815 & 5.012 \\
\hline \multirow{2}{*}{$\begin{array}{l}\text { SET with } \\
\text { filter } \\
\text { failure }\end{array}$} & $\begin{array}{c}\text { Rényi } \\
\text { entropy }\end{array}$ & 12.541 & 12.984 & 13.841 & 12.741 \\
\hline & $\begin{array}{l}\text { Estimation } \\
\text { error(Hz) }\end{array}$ & 1.045 & 1.195 & 1.684 & 1.121 \\
\hline
\end{tabular}

It can be seen from Fig. 9 and Table 3 that in case of severe fault, the SNR of the vibration signal is large, and the fault information is more obvious than the noise information. Therefore, the Rényi entropy and estimation error based on the proposed algorithm are smaller, so it can be seen that the time-frequency concentration and accuracy of the algorithm are higher. However, with the reduction of fault degree, the SNR of the vibration signal is reduced, and the noise is more obvious than the fault information, so the time-frequency concentration and accuracy of the algorithm are reduced. By analyzing the size of the fault, it can be seen that the actual fault corresponds to the moderate fault, and by comparing the performance index of the test results, it is found that the test results of the actual fault are most similar to that of the moderate fault. So the test results are consistent with the theoretical analysis. Moreover, compared with SST and STFT, the time-frequency concentration and estimation accuracy of the proposed algorithm are still significantly improved. Therefore, the proposed algorithm is suitable for fault diagnosis of the rolling bearing with different fault degrees. The introduction of the simulation results of the actual fault also proves that the proposed algorithm is not only suitable for uniform processing faults, but also has a good effect on non-uniform actual faults.

\subsection{Fault detection in fluctuated speed condition}

The data under complex fluctuated speed conditions is very large. This article intercepts part of them for analysis. The vibration signal waveform is shown in Fig. 10(a) which is accompanied by a lot of noise and the simulation test is carried out for the moderate failure of the outer race whose fault size is $4 \mathrm{~mm} \times 0.5 \mathrm{~mm} \times 0.5 \mathrm{~mm}$. The rotational frequency varies from $6 \mathrm{~Hz}$ to $20 \mathrm{~Hz}$. According to Eq. (16), the fault characteristic frequency bandwidth of vibration is from $31.404 \mathrm{~Hz}$ to $104.68 \mathrm{~Hz}$. The Fourier spectrum is shown in Fig. 10(b). Choose the optimal filter band with fast spectral kurtosis, the fast kurtogram is shown in Fig. 10(c). From kurtogram it can be determined that the optimal bandwidth is $800 \mathrm{~Hz}$ and the center frequency is $3175 \mathrm{~Hz}$. Then, the amplitude envelope of filtered signal is shown in Fig. 10(d). At last, perform SET on amplitude envelope, the result is shown in Fig. 10(e). From the time-frequency representation, we can see that SET can accurately extract fault characteristic frequency, and has high time-frequency concentration, and is basically free from noise interference. According to time-frequency representation of SET, we can determine that the outer race exists fault. Figures 10(f) and (g) are the results of SST and STFT, respectively. By comparison, it can be seen that under complicated noise interference, SST can't extract fault characteristic frequency. There are too many uncorrelated frequency components in the STFT time-frequency demodulation spectrum, and the time-frequency energy spreads seriously. Figure 10(h) shows the result of SET without filter, it can be seen that it can still clearly demodulate fault characteristic frequency. The time-frequency concentrations of the SET, SST, STFT and SET with filter failure are analyzed by the Rényi entropy, and the results are shown in Table 4. According to the comparison in Table 4, the Rényi entropy of the SET is the smallest and the time-frequency concentration is the highest. Even if the filter fails, the Rényi entropy of the algorithm is still less than SST and STFT. Therefore, the envelope demodulation method based on SET not only improves the time-frequency concentration of the algorithm, but 
also has strong robustness and anti-interference ability.

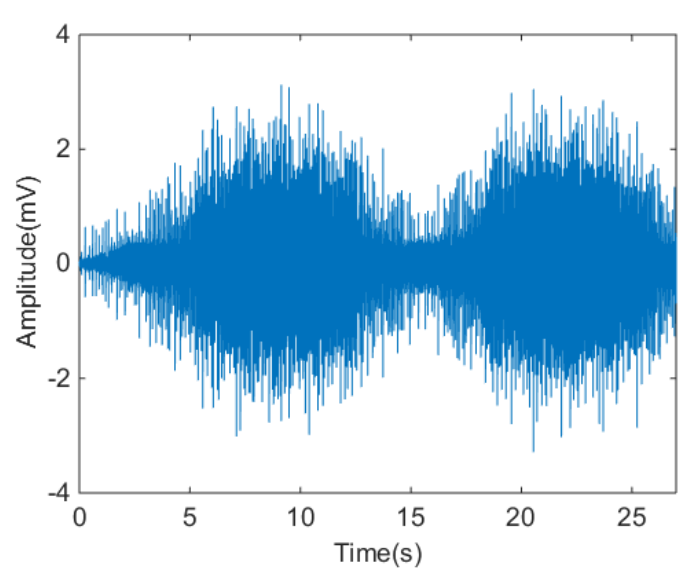

(a) The time domain diagram

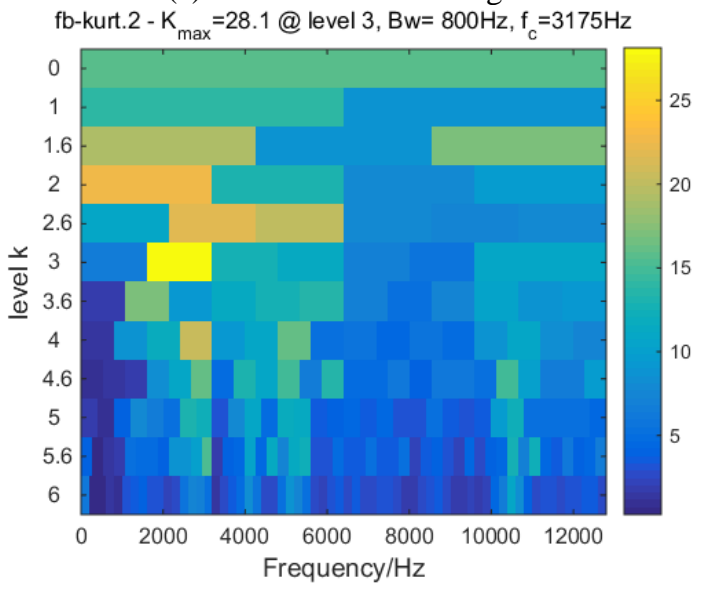

(c) The kurtogram

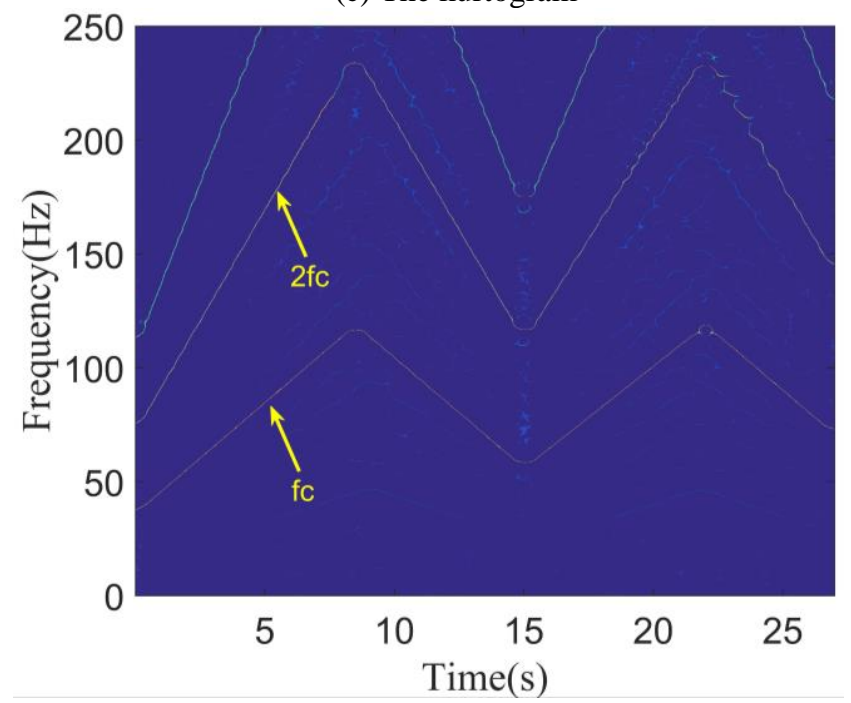

(e) SET

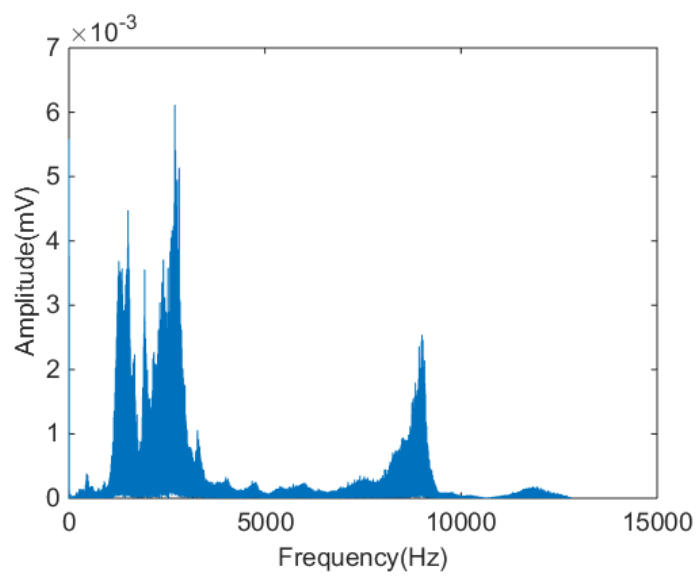

(b) The frequency domain waveform

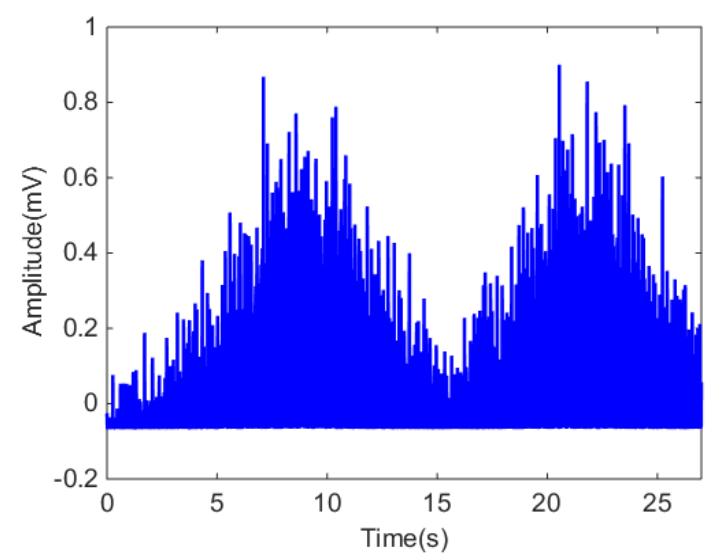

(d) The amplitude envelope

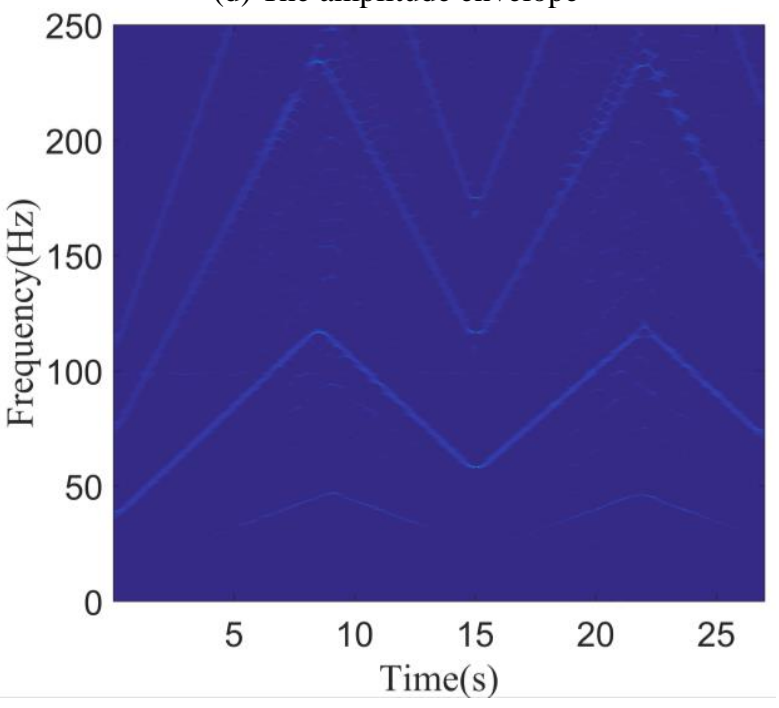

(f) SST 


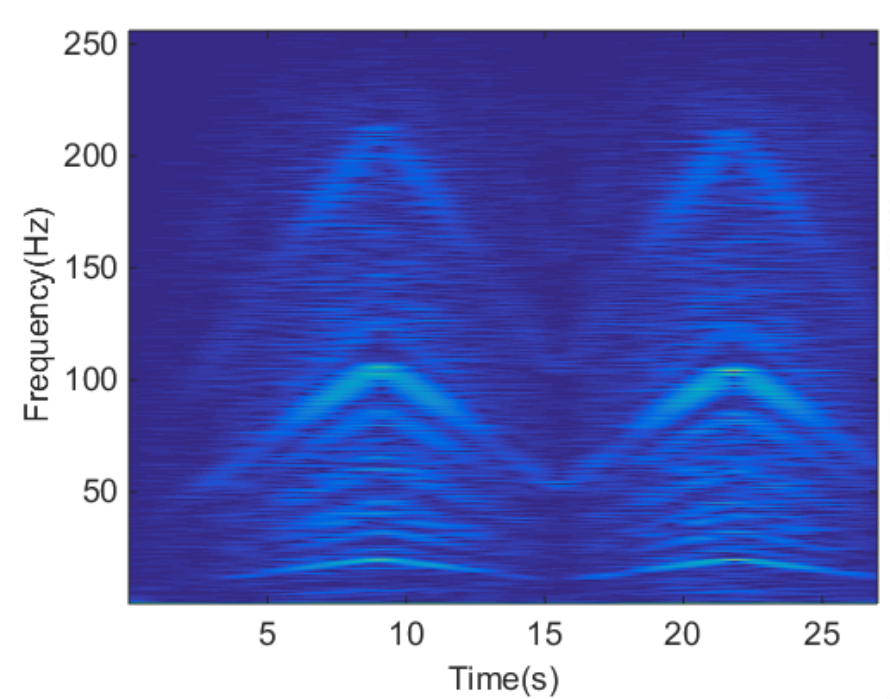

(g) STFT

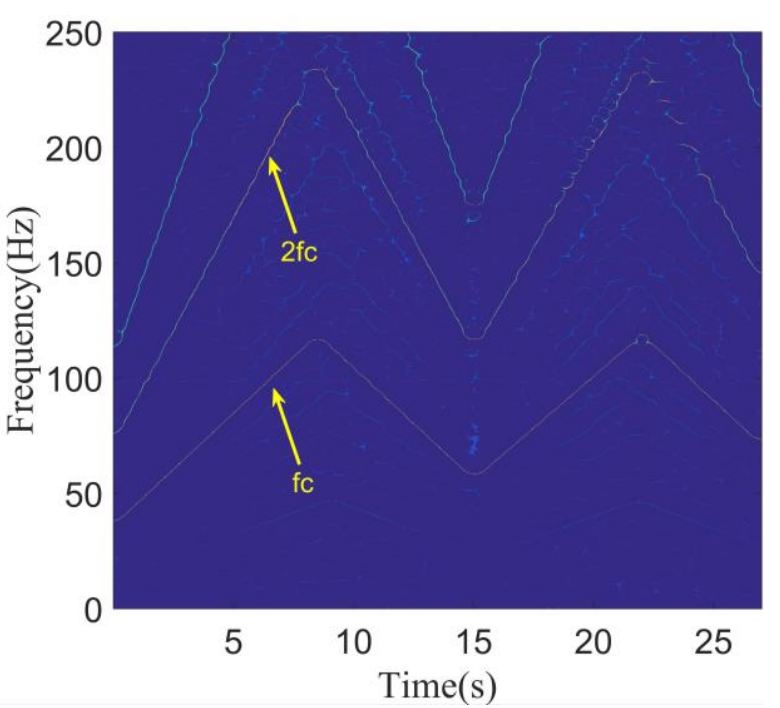

(h) SET with filter failure

Fig. 10 Real signal analysis results under fluctuated speed.

Table 4 The Rényi entropy

\begin{tabular}{c|c}
\hline \hline Algorithms & Rényi entropy \\
\hline SET & 14.052 \\
\hline SST & 16.215 \\
\hline STFT & 19.954 \\
\hline SET with filter failure & 14.854 \\
\hline
\end{tabular}

\section{Conclusion}

In order to solve the problem of rolling bearing fault diagnosis under variable speed condition, the envelope demodulation time-frequency analysis method based on SET is proposed. Firstly, the band-pass filtering based on fast spectral kurtosis is performed to select the fault-sensitive components. Then calculate amplitude envelope of the filtered signal by Hilbert transform. Finally, SET is used to perform time-frequency demodulation spectrum on the amplitude envelope. The simulation and comparison of the proposed method are carried out by using the simulation signals and the actual signals, and some conclusions are as follows:

(i). The high time-frequency concentration. Compared with the SST and STFT, the proposed method improves the energy concentration of the time-frequency distribution greatly. For simulation signals and actual signals, the Rényi entropy of the proposed algorithm is less than SST and STFT, as shown in Fig. 4 and Table 3.

(ii). Noise robustness. With the increase of the SNR, the Rényi entropy of the proposed method does not fluctuate and it is always less than SST and STFT. Hence, it can be seen that the proposed method has strong noise robustness and is more suitable for practical engineering applications.

(iii). The Robustness with band-pass filter failure. In the case of failure of the band-pass filter, the proposed method can still suppress noise interference and clearly extract the fault characteristic frequency, which is still consistent with the theoretical fault characteristic frequency. The result proves that the proposed method has good robustness and anti-interference property, and it still works well when filter fails suddenly.

(iv). The high estimation accuracy of fault characteristic frequency. Compared with the SST and STFT under rising speed, the estimation error of fault characteristic frequency based on the proposed method is the least for the four fault degree. On average, the estimation error is $2.1833 \mathrm{~Hz}$ less than the corresponding error of the SST and $5.0087 \mathrm{~Hz}$ less than the corresponding error of the STFT method.

\section{Acknowledgments}

This research is supported by the National Natural Science Foundation of China (Grant No. 11790282), the 2020 Independent Project of State Key Laboratory of Mechanical Behavior and System Safety of Traffic Engineering Structures (Grant No. ZZ2020-39), the Hebei Province “333” Talents Project (Grant No. A201802004), the Graduate 
Innovation Funding Project of Hebei Province (Grant No. CXZZBS2019152), and the 2020 Graduate Innovation Funding Project (Grant No. YC2020062). The authors gratefully acknowledge these support agencies.

\section{References}

Amstrup, S. C., Mcdonald, T. L., and Durner G. M., Using satellite radiotelemetry data to delineate and manage wildlife populations, Wildlife Society Bulletin, Vol.32, No.3 (2004),pp. 661-679.

Bin J., Zhang H. J., Liu Y. B., Liu F., Lu S. L., and Dai Z. J., A feature extraction method using improved multi-scale entropy for rolling bearing fault diagnosis, Entropy, Vol.20, No.4 (2018), pp. 212.

Bo G., Xu G. J., Liu X. F., and Lin J., Bearing Fault Diagnosis based on Subband Time-Frequency Texture Tensor, IEEE Access, Vol.7 (2019), pp. 37611-37619.

Borghesani P., Pennacchi P., and Chatterton S., The relationship between kurtosis-and envelope-based indexes for the diagnostic of rolling element bearings, Mechanical Systems \& Signal Processing, Vol.43 (2014),pp.25-43.

Chen B. J., Shen B. M., Chen F. F., Tian H. L., Xiao W. R., Zhang F. J., and Zhao C. H., Fault diagnosis method based on integration of RSSD and wavelet transform to rolling bearing, Measurement, Vol.131 (2019), pp.400-411.

Chen H., Kang J. X., Chen Y. C., Xu D., and Hu Y., An Improved Time-Frequency Analysis Method for Hydrocarbon Detection Based on EWT and SET, Energies, Vol.10 ( 2017), pp. 1090.

Chen Z. G., Zhai W. M., and Wang K. Y., Vibration feature evolution of locomotive with tooth root crack propagation of gear transmission system, Mechanical Systems \& Signal Processing, Vol.115 (2019), pp.29-44.

Claasm T. A. C. M., and Mecklenbrauker W. F. G. The wigner distribution-a tool for time-frequency signal analysis, Philips Journal of Research, Vol.35, No.(4-5) (1980), pp. 276-300.

Daubechies I., Lu F. J., and Wu H. T., Synchrosqueezed wavelet transforms: An empirical mode decomposition-like tool. Applied and Computational Harmonic Analysis, Vol.30, No.2 (2011), pp.243-261.

Daubechies I., Wang Y., and Wu H. T., ConceFT: concentration of frequency and time via a multitapered synchrosqueezed transform, Philosophical Transactions of the Royal Society A: Mathematical, Physical and Engineering Sciences (online), available from < https://doi.org/10.1098/rsta.2015.0193>, (2016).

Feng Z. P., Chen X. W., and Liang M., Iterative generalized synchrosqueezing transform for fault diagnosis of wind turbine planetary gearbox under nonstationary conditions, Mechanical Systems \& Signal Processing, Vol.52-53 (2015), pp. 360-375.

Feng Z P., Chen X. W., and Wang T. Y., Time-varying demodulation analysis for rolling bearing fault diagnosis under variable speed conditions, Journal of Sound Vibration, Vol.400 (2017), pp. 71-85.

Gao Y. Y., Yu D. J., and Wang H. J., Fault diagnosis of rolling bearings using weighted horizontal visibility graph and graph Fourier transform, Measurement, Vol.149 (2020), pp. 107036.

Glowacz A., Glowacz W., Glowacz Z., and Kozik J., Early fault diagnosis of bearing and stator faults of the single-phase induction motor using acoustic signals , Measurement, Vol.113 (2018), pp.1-9.

Hoseinzadeh M. S., Khadem S. E., and Sadooghi M. S., Modifying the Hilbert-Huang transform using the nonlinear entropy-based features for early fault detection of ball bearings, Applied Acoustics, Vol.150 (2019), pp.313-324.

Li C., and Liang M., Time-frequency signal analysis for gearbox fault diagnosis using a generalized synchrosqueezing transform, Mechanical Systems \& Signal Processing, Vol.26 (2012), pp.205-217.

Li X., Ma Z. Q., Kang D., and Li X., Fault diagnosis for rolling bearing based on VMD-FRFT, Measurement, Vol.155 (2020), pp.107544.

Liu D. D., Cheng W. D., and Wen W. G., Rolling Bearing Fault Diagnosis via ConceFT-Based Time-Frequency Reconfiguration Order Spectrum Analysis, IEEE Access, Vol.6 (2018), pp. 67131-67143.

Liu M. K., and Weng P. Y., Fault Diagnosis of Ball Bearing Elements: A Generic Procedure based on Time-Frequency Analysis, Measurement Science Review, Vol.19, No.4 (2019), pp.185-194.

Ma Z. Q., Lu F. Y., Liu S. Y., and Li X., An Adaptive Generalized Demodulation Method for Multimedia Spectrum Analysis is Applied in Rolling Bearing Fault Diagnosis, IEEE Access, Vol.8 (2020), pp. 20687-20699.

Mustafa M., Abdul R. A., Srete N., Tole S., and Jopri M.H., An improved smooth-windowed wigner-ville distribution analysis for voltage variation signal, International Journal of Electrical and Computer Engineering, Vol.10, No.5 (2020), pp. 4982-4991. 
Qin C. R., Wang D. D., Xu Z., and Tang G., Improved Empirical Wavelet Transform for Compound Weak Bearing Fault Diagnosis with Acoustic Signals, Applied Sciences, Vol.10, No. 2 (2020), pp.682.

Tao H. F., Wang P., and Chen Y. Y., Vladimir Stojanovicc; Huizhong Yang. An unsupervised fault diagnosis method for rolling bearing using STFT and generative neural networks, Journal of the Franklin Institute (online), available from < https://doi.org/10.1016/j.jfranklin.2020.04.024>, (2020).

Wang S. B., Chen X. F., Selesnick I. W., Guo Y. J., Tong C. W., and Zhang X. W., Matching synchrosqueezing transform: A useful tool for characterizing signals with fast varying instantaneous frequency and application to machine fault diagnosis, Mechanical Systems \& Signal Processing. Vol. 100 (2018), pp.242-288.

Wang T. Y., Lan Z., Qiao H. H., and Wang P., Fault diagnosis of rotating machinery under time-varying speed based on order tracking and deep learning, Journal of Vibroengineering, Vol.22, No.2 (2018), pp.366-382.

Wu J., Zi Y. Y., Chen J. L., and Zhou Z. T., Fault diagnosis in speed variation conditions via improved tacholess order tracking technique, Measurement, Vol.137 (2019), pp.604-616.

Yu G., Research on excavator vibration and sound signal processing using time-frequency analysis method and implementation, Dissertation for Doctoral Degree, Shandong University, Shandong, China, 2016.

Yu G., Yu M., and Xu C. Y., Synchroextracting Transform, IEEE Transactions on Industrial Electronics, Vol.64, No.10 (2017), pp.8042-8054.

Zoltan G. S., and Horatiu S. G., Hilbert-Huang Transform in Fault Detection, Procedia Manufacturing, Vol.32 (2019), pp. 591-595.

Zou H. X., Dai H. Q., Li Y. D., and Lu X. G., The Nonexistence of Time-frequency Distribution with WVD Aggregation and without Cross Terms, Scientia Sinica(Technologica), Vol.31 ( 2001), pp. 348-354. (in Chinese) 